\title{
Interpolative Boolean Algebra for Generalizations of Intuitionistic Fuzzy Sets
}

\author{
Pavle Milošević ${ }^{a}$ and Bratislav Petrović ${ }^{b}$ \\ ${ }^{\mathrm{a}}$ Department of Systems Theory and Control, Faculty of Organizational Sciences, University of Belgrade, Jove \\ Ilića 154, Belgrade, 11000, Serbia, pavle.milosevic@fon.bg.ac.rs \\ ${ }^{b}$ Department of Systems Theory and Control, Faculty of Organizational Sciences, University of Belgrade, Jove \\ Ilića 154, Belgrade, 11000, Serbia, bratislav.petrovic@fon.bg.ac.rs
}

\begin{abstract}
The idea of this paper is to investigate intuitionistic fuzzy set (IFS) generalizations and make one step further into that direction by introducing interpolative Boolean algebra (IBA) to Liu's generalized IFS. First, we aim to give an overview of the existing attempts to generalize or alter the definition of IFS and to underline the most general approach. Further, Liu's bipolar I-fuzzy sets (LBIFSs) are introduced as the most universal case of Liu's generalized intuitionistic fuzzy sets. Finally, IBA-based operations are introduced to deal with LBIFS. In the proposed LBIFS-IBA approach, all Boolean laws are fulfilled. The special attention is dedicated to laws of the excluded middle and contradiction.
\end{abstract}

Keywords: Intuitionistic fuzzy sets, Interpolative Boolean algebra, Generalizations of intuitionistic fuzzy sets, Liu's generalized IFS, IFS-interpretational triangle.

\section{Introduction}

In the presence of imprecise information it is a challenging task to comprehend and present information in a proper manner, and further to use it to make good decisions. Intuitionistic fuzzy set (IFS) theory, proposed by Atanassov [1], is the valuable tool for information presentation and manipulation. IFSs are considered as a generalization of traditional fuzzy sets, by incorporating not only degree of membership, but also degrees of non-membership and non-determinacy (uncertainty). Operations and relations over IFSs extend the relations and operations over fuzzy sets [3].
As the one of the many extensions of fuzzy sets, IFS are thoroughly studied from both theoretical (e.g. [2, $3,5]$ ) and practical point of view (e.g. $[11,12,14,23$, 24]). Further, IFS are used as a basis for different enhancements, such as interval valued IFS [4]. However, in this paper, we will focus only on the variations and generalizations of IFS definitions in the sense of IFS-interpretational surface. Consequently, we will also reflect on the problems of estimating degrees of membership and nonmembership to a certain IFS [3].

Interpolative Boolean algebra (IBA) is a consistent [0,1]-valued generalization of classical Boolean logic [19]. IBA is introduced in IFS theory in IFS-IBA approach [16]. On the one hand, it has brought the desired mathematical properties to the approach, and on the other, provided flexibility since various $t$-norm operators may be applied as operators.

In this paper, we aim to define a generalization of IFS - Liu's bipolar I-fuzzy sets (LBIFS) as a special case of Liu's intuitionistic fuzzy sets [13]. LBIFS extends classical IFS-interpretational triangle to interpretational square, enabling more descriptive power. Further, we introduce IBA-based operations over LBIFS. Finally, we show that the operations defined in the proposed LBIFS-IBA approach satisfy Boolean laws. The special attention is devoted to the validity of the laws of excluded middle and contradiction.

This paper is organized as follows. The basic notation and the most prominent generalizations of IFS are listed in Section 2. In Section 3 the brief overview of IBA is given. The introduction of IBAbased operations on generalized IFS is presented in Section 4. Finally, the main conclusions and potential directions of future work are presented in Section 5.

\section{Generalizations of intuitionistic fuzzy sets}

Intuitionistic fuzzy set theory is a well-known generalization of the traditional fuzzy set theory [3]. IFSs take into account both membership and nonmembership of an element to a certain set providing 
the greater description power comparing to traditional fuzzy sets. In literature, IFS are often called Atanassov IFS.

Definition 1 [1]. An intuitionistic fuzzy set $A$ in a universe $X$ is defined as an object:

$$
A=\left\{\left(x, \mu_{A}(x), v_{A}(x) \mid x \in X\right)\right\}=\left\langle\mu_{A}, v_{A}\right\rangle
$$

where functions $\quad \mu_{A}(x): X \rightarrow[0,1] \quad$ and $v_{A}(x): X \rightarrow[0,1]$ represent degrees of membership and non-membership of the element $x$ to the IFS $A$, and for every $x \in X$ the sum of degrees of membership and non-membership is:

$0 \leq \mu_{A}+v_{A} \leq 1$.

The definition of IFS implicitly includes the existence of a certain level of uncertainty (the degree of non-determinacy) $\pi_{A}(x)$ of the element $x \in E$ to

$A$, since:

$\pi_{A}=1-\left(\mu_{A}+v_{A}\right)$.

Each IFS may be graphically represented within IFSinterpretational triangle [3], given in Figure 1.

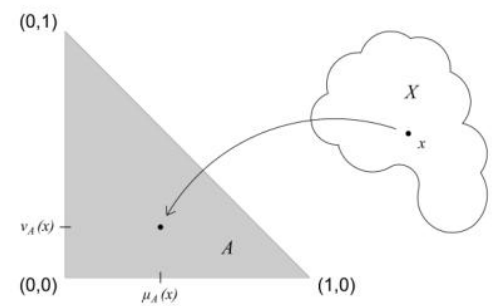

Figure 1: IFS-interpretational triangle

IFS in the vertex $(0,0)$ is totally undefined, since its degree of non-determinacy is equal to 1 . Vertices $(0,1)$ and $(1,0)$ represent special cases of IFSs that are reduced to classical crisp sets. Further, IFSs that are on the hypotenuse of IFS-interpretational triangle may be observed as classical fuzzy sets since these in cases there is no uncertainty $\left(\mu_{A}+v_{A}=1, \pi_{A}=0\right)$.

One of the main issues in IFS theory is possibility to extend the definition of IFS in terms of IFSinterpretational triangle. There are numerous attempts to extend interpretational surface or to establish addition dependence between membership and non-membership degree.

The approach proposed in [17] suggests including a restriction of logical dependence between membership and non-membership degree.
Definition 2 [17]. A generalized intuitionistic fuzzy set $A^{M S}$ in a universe $X$ in the sense of [17] is defined as an object:

$$
A^{M S}=\left\{\left(x, \mu_{A}^{M S}(x), v_{A}^{M S}(x) \mid x \in X\right)\right\}=\left\langle\mu_{A}^{M S}, v_{A}^{M S}\right\rangle,
$$

where:

$$
0 \leq \mu_{A}^{M S} \wedge v_{A}^{M S} \leq 0.5, \quad \mu_{A}^{M S}(x), v_{A}^{M S}(x): X \rightarrow[0,1] .
$$

In other words, at least one of the degrees of membership and non-membership should be less than 0.5 if minimum is applied as $t$-norm. Therefore, $A^{M S}=\left\langle\mu_{A}^{M S}, v_{A}^{M S}\right\rangle=\langle 0.8,0.4\rangle$ is valid generalized IFS in the sense of [17], while it is not IFS.

In [7], authors introduced 7 various generalizations and/or alternations of IFS definition, along with the suitable graphical interpretation ${ }^{1}$. One of the proposed IFS alternations is given in the following definition and illustrated in Figure 2.

Definition 3 [7]. A generalized intuitionistic fuzzy set $A^{D O J}$ in a universe $X$ in the sense of GAIFS5 in [7] is defined as an object:

$$
A^{D O J}=\left\{\left(x, \mu_{A}^{D O J}(x), v_{A}^{D O J}(x) \mid x \in X\right)\right\}=\left\langle\mu_{A}^{D O J}, v_{A}^{D O J}\right\rangle,
$$

where:

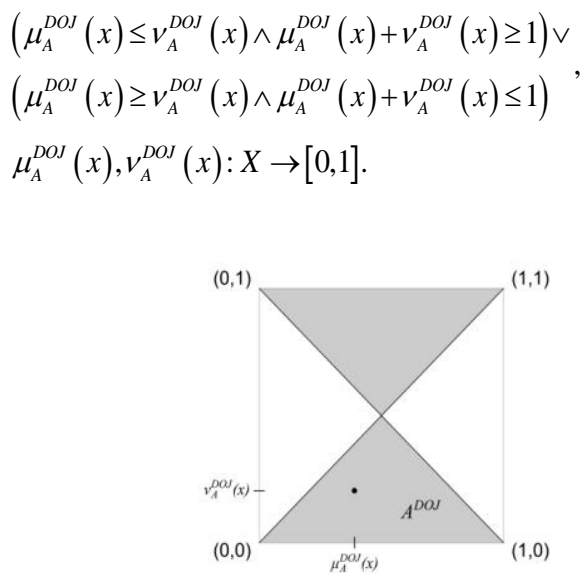

Figure 2: Interpretational surface of generalized IFS in the sense of GAIFS5 in [7]

It should be noticed that of the approaches in [7], IFS of root type, is proposed parallelly in [22].

In [10], authors parameterize definitions of root type IFS proposed in [7, 22].

\footnotetext{
${ }^{1}$ Graphical interpretation of one of the generalization (GAIFS6) given in [7] is not correct. The correct graphical interpretation may be found in [10].
} 
Definition 4 [10]. A generalized intuitionistic fuzzy set $A^{J N}$ in a universe $X$ in the sense of [10] is defined as an object:

$A^{J N}=\left\{\left(x, \mu_{A}^{J N}(x), v_{A}^{J N}(x) \mid x \in X\right)\right\}=\left\langle\mu_{A}^{J N}, v_{A}^{J N}\right\rangle$,

where:

$0 \leq\left(\mu_{A}^{J N}\right)^{\delta}+\left(v_{A}^{J N}\right)^{\delta} \leq 1, \quad \mu_{A}^{J N}(x), v_{A}^{J N}(x): X \rightarrow[0,1]$,

and $\delta=n \vee 1 / n, n=1, \ldots, N$.

In the case when $\delta=1, A^{J N}$ is classical IFS, while for $\delta=2, A^{J N}$ is root type IFS.

Another parameterization of IFS definition is proposed in [13]. The main idea of the paper is to extend IFS-interpretational surface by introducing parameter $L$.

Definition 5 [13]. Liu's generalized intuitionistic fuzzy set $A^{L}$ in a universe $X$ is defined as an object:

$A^{L}=\left\{\left(x, \mu_{A}^{L}(x), v_{A}^{L}(x) \mid x \in X\right)\right\}=\left\langle\mu_{A}^{L}, v_{A}^{L}\right\rangle$,

where:

$0 \leq \mu_{A}^{L}+v_{A}^{L} \leq 1+L, \quad \mu_{A}^{L}(x), v_{A}^{L}(x): X \rightarrow[0,1]$,

and $L \in[0,1]$.

The example of Liu's generalized IFSinterpretational surface for $L=0.6$ is presented in Figure 3.

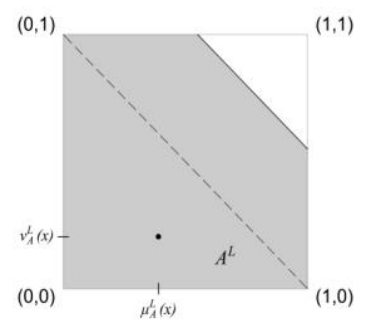

Figure 3: Interpretational surface of Liu's generalized IFS for $L=0.6$

Liu's generalized IFS offers the greatest generalization power for $L=1$, transforming interpretational surface to square. This special case of Liu's generalized IFS may be observed as the most general among the listed approaches.

Operations on generalized IFSs are realized in a same manner as classical IFS operations. Therefore, the algebra on generalized IFS has same properties as IF algebra.

Although the most of IFS generalizations are intuitive and mathematically correct, the question of practical justification and applicability of the proposed approaches arises. The most of the papers on this topic exclusively provide a mathematical apparatus for generalization, but not an explanation of why generalization is needed; neither provides necessary interpretation nor the application guidelines. However, there are several papers dealing with the application of listed approaches, mainly for similarity measuring $[6,18]$.

\section{Interpolative Boolean algebra}

Interpolative Boolean algebra is a consistent generalization of classical Boolean algebra in the sense that all elements of Boolean algebra $B A(\Omega)$ are realized in $[0,1]$ interval [19]. In IBA, all Boolean axioms are preserved and used as a basis for Boolean consistent fuzzy logic [20] and IFS-IBA approach [16]. By distinguishing the symbolic and valued level, IBA focuses on the structure instead on the values as in conventional multi-valued algebras [20].

On the symbolic IBA level, each logical function is transformed to generalized Boolean polynomial (GBP), i.e. a polynomial that consists of BA elements as variables, and generalized product $(\otimes)$, standard plus and minus as operators. Complex logical expressions (i.e. combined elements of BA) $F\left(p_{1}, \ldots, p_{n}\right), G\left(p_{1}, \ldots, p_{n}\right) \in B A(\boldsymbol{\Omega})$ are mapped to GBP in the following manner:

$$
\begin{aligned}
& (F \wedge G)^{\otimes}=F^{\otimes} \otimes G^{\otimes}, \\
& (F \vee G)^{\otimes}=F^{\otimes}+G^{\otimes}-F^{\otimes} \otimes G^{\otimes}, \\
& (\neg F)^{\otimes}=1-F^{\otimes} .
\end{aligned}
$$

On the other hand, the rules for transforming primary elements $\boldsymbol{\Omega} \in\left\{p_{1}, \ldots, p_{n}\right\}$ are:

$$
\begin{aligned}
& \left(p_{i} \wedge p_{j}\right)^{\otimes}=\left\{\begin{array}{ll}
p_{i} \otimes p_{j}, & i \neq j \\
p_{i}, & i=j
\end{array},\right. \\
& \left(p_{i} \vee p_{j}\right)^{\otimes}=p_{i}+p_{j}-\left(p_{i} \wedge p_{j}\right)^{\otimes}, \\
& \left(\neg p_{i}\right)^{\otimes}=1-p_{i}^{\otimes} .
\end{aligned}
$$

The valued level is introduced after the transformation procedure has been completed. All primary elements in the obtained GBP are valued in a $[0,1]$ interval, and suitable operator for generalized product is selected. Generalized product is a subclass of $t$-norms; i.e. it satisfies all four axioms of $t$-norms (commutativity, associativity, monotonicity, boundary condition), and the additional axiom - nonnegativity condition [21]. The choice of generalized product is based on the nature of the $B A(\Omega)$ elements and/or correlation between them [8, 15]. For instance, minimum should be used for elements of the same/similar nature. For the elements that are 
statistically independent (i.e. different by nature) standard product should be utilized.

\section{IBA for generalized IFS}

In this section, we aim to introduce IBA-based operation on the generalized IFS. In more details, we utilize pairs of generalized Boolean polynomials to realize logical operations on the most comprehensive form of Liu's IFS.

Liu's bipolar I-fuzzy set. Since we will focus on Boolean properties of the proposed approach, it is not appropriate to name the approach 'intuitionistic'. Therefore, we introduce a notion of Liu's bipolar Ifuzzy set (LBIFS) as a special case of Liu's generalized intuitionistic fuzzy set when $L=1$. Bipolar I-fuzzy set was proposed as an alternative term for IFS during the terminological debate [9] and it seems to be suitable in this case.

Definition 6. Liu's bipolar I-fuzzy set $A^{B}$ in a universe $X$ is defined as an object:

$A^{B}=\left\{\left(x, \mu_{A}^{B}(x), v_{A}^{B}(x) \mid x \in X\right)\right\}=\left\langle\mu_{A}^{B}, v_{A}^{B}\right\rangle$,

where:

$0 \leq \mu_{A}^{B}+v_{A}^{B} \leq 2, \quad \mu_{A}^{B}(x), v_{A}^{B}(x): X \rightarrow[0,1]$.

The uncertainty (the degree of non-determinacy) $\pi_{A}^{B}(x)$ of the element $x \in E$ to LBIFS $A^{B}$, is calculated in the same manner as in IFS theory:

$\pi_{A}=1-\left(\mu_{A}+v_{A}\right)$.

The example of LBIFS is presented in Figure 4.

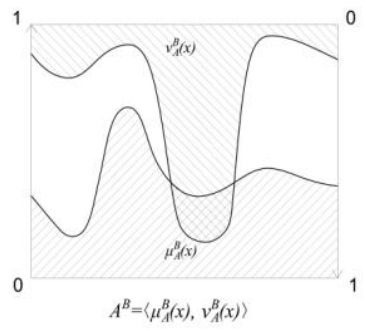

Figure 4: Graphical interpretation of LBIFS $A^{B}$

LBIFS may be seen as another way to deal with problems in relation to the correctness of the expert estimations of IFS. In practice, experts commonly violate $\mu_{A}+v_{A} \leq 1$ condition when estimating degrees of membership and non-membership to a certain IFS [3]. Incorrect estimations of $\mu_{A}$ and $v_{A}$ are transformed in valid IFS using linear transformations that usually alter estimations significantly. Assuming that an expert gave values to $\mu_{A}$ and $v_{A}$ with intention, LBIFS may properly deal with incorrect estimations in the sense of IFS capturing the essence of the estimation simultaneously.

LBIFS-interpretational square is given in Figure 5. This surface extends classical IFS-interpretational triangle.

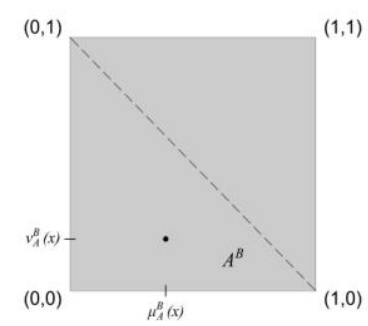

Figure 5: LBIFS-interpretational square

LBIFSs in the vertices $(0,0)$ and $(1,1)$ are totally undefined, since the absolute value of uncertainty is equal to 1 in both cases. However, these LBIFS differs in meaning since the uncertainty differs in sign. Therefore, the uncertainty of LBIFS may be interpreted in the similar as Pearson correlation coefficient: the absolute value of the uncertainty depicts the level of uncertainty, while the sign explains the origin (i.e. whether functions of membership and non-membership overlap or not).

IBA-based operations over LBIFS. The IBA-based operations of conjunction, disjunction and negation (intersection, union and complement in set notation) over LBIFS are defined in the following manner:

$$
\begin{aligned}
& \left(A^{B} \wedge B^{B}\right)^{\otimes}=\left\langle\mu_{A}^{B} \otimes \mu_{B}^{B}, v_{A}^{B}+v_{A}^{B}-v_{A}^{B} \otimes v_{A}^{B}\right\rangle, \\
& \left(A^{B} \vee B^{B}\right)^{\otimes}=\left\langle\mu_{A}^{B}+\mu_{B}^{B}-\mu_{A}^{B} \otimes \mu_{B}^{B}, v_{A}^{B} \otimes v_{A}^{B}\right\rangle, \\
& \left(\neg A^{B}\right)^{\otimes}=\left\langle 1-\mu_{A}^{B}, 1-v_{A}^{B}\right\rangle .
\end{aligned}
$$

In case of complex logical expressions, IBA transformation rules should be applied.

Operations of conjunction and disjunction are realized in the same manner as in IBA-IFS approach $[14,16]$ and they may be perceived as analogous to the conventional IF operation. The operation of negation is derived on the basis of negation operators for classical fuzzy sets. The proposed operator is not valid in IFS framework, since:

$1-\mu_{A}^{B}+1-v_{A}^{B}=2-\left(\mu_{A}^{B}+v_{A}^{B}\right) \leq 1$

stands only for a classical fuzzy set $\left(\mu_{A}+v_{A}=1\right)$. However, this negation is valid for LBIFS. It implies that for starting LBIFS $A^{B}$, the uncertainty of its 
negation $\neg A^{B}$ is same in absolute value, but different in the sign except in the case of classical fuzzy set:

$$
\begin{aligned}
& 1-\left(\mu_{A}^{B}+v_{A}^{B}\right)=\pi_{A}^{B} \\
& 1-\left(1-\mu_{A}^{B}+1-v_{A}^{B}\right)=-1+\left(\mu_{A}^{B}+v_{A}^{B}\right)=-1+\left(\mu_{A}^{B}+v_{A}^{B}\right)=-\pi_{A}^{B} .
\end{aligned}
$$

The proposed negation satisfies the double negation rule, i.e. it is an involutive negation operator, since:

$$
\left(\neg\left(\neg A^{B}\right)\right)^{\otimes}=\left\langle 1-\left(1-\mu_{A}^{B}\right), 1-\left(1-v_{A}^{B}\right)\right\rangle=\left\langle\mu_{A}^{B}, v_{A}^{B}\right\rangle .
$$

Boolean properties of LBIFS-IBA approach. Since IBA-based operations and transformation rules are directly applied for dealing with LBIFS, all Boolean properties, including the laws of contradiction and excluded middle, are satisfied in general case. These two Boolean laws are of a particular significance in the area intuitionistic, fuzzy and IF logic and it is often a theme for debate. Therefore, the validity of contradiction and excluded middle is investigated in details.

In IFS theory, the validity of the law of excluded middle is studied in four forms: 1) tautology form; 2) intuitionistic fuzzy tautology (IFT) form; 3) modified law of excluded middle in tautology form; 4) modified law of excluded middle in IFT form [3]. However, in this paper, we consider only the classical tautology form, since it assumes the strongest condition.

$$
\begin{aligned}
\left(A^{B} \vee \neg A^{B}\right)^{\otimes} & =\left\langle\mu_{A}^{B}, v_{A}^{B}\right\rangle \vee\left\langle 1-\mu_{A}^{B}, 1-v_{A}^{B}\right\rangle \\
& =\left\langle\mu_{A}^{B}+1-\mu_{A}^{B}-\mu_{A}^{B} \otimes\left(1-\mu_{A}^{B}\right), v_{A}^{B} \otimes\left(1-v_{A}^{B}\right)\right\rangle= \\
& =\left\langle\mu_{A}^{B}+1-\mu_{A}^{B}-\mu_{A}^{B}+\mu_{A}^{B} \otimes \mu_{A}^{B}, v_{A}^{B}-v_{A}^{B} \otimes v_{A}^{B}\right\rangle= \\
& =\left\langle 1-\mu_{A}^{B}+\mu_{A}^{B}, v_{A}^{B}-v_{A}^{B}\right\rangle= \\
& \equiv\langle 1,0\rangle
\end{aligned}
$$

As it is shown, the law of excluded middle is a tautology in the proposed approach.

The law of contradiction is also studied in the tautology form.

$$
\begin{aligned}
\left(A^{B} \wedge \neg A^{B}\right)^{\otimes} & =\left\langle\mu_{A}^{B}, v_{A}^{B}\right\rangle \wedge\left\langle 1-\mu_{A}^{B}, 1-v_{A}^{B}\right\rangle \\
& =\left\langle\mu_{A}^{B} \otimes\left(1-\mu_{A}^{B}\right), v_{A}^{B}+1-v_{A}^{B}-v_{A}^{B} \otimes\left(1-v_{A}^{B}\right)\right\rangle= \\
& =\left\langle\mu_{A}^{B}-\mu_{A}^{B} \otimes \mu_{A}^{B}, v_{A}^{B}+1-v_{A}^{B}-v_{A}^{B}+v_{A}^{B} \otimes v_{A}^{B}\right\rangle= \\
& =\left\langle\mu_{A}^{B}-\mu_{A}^{B}, 1-v_{A}^{B}+v_{A}^{B}\right\rangle= \\
& \equiv\langle 0,1\rangle
\end{aligned}
$$

Therefore, it is proven that the law of contradiction is valid in LBIFS-IBA approach.

\section{Conclusion}

In the last decades, generalizing the definition of classical fuzzy sets as well as IFSs is very important and actual topic. Authors are mainly concentrated on altering the definition of IFS in order to achieve more descriptive power, while the interpretability and operations over generalized IFSs are not in the focus.

In this paper, we have introduced LBIFS-IBA approach. More precisely, we have defined logical operations based on IBA over generalized IFS, Liu's bipolar I-fuzzy sets. LBIFS is a special case of Liu's generalized IFS with the greatest descriptive power, extending IFS-interpretational triangle to LBIFSinterpretational square. On the other hand, IBA-based operations of conjunction and disjunction are defined in the same manner as in IFS-IBA approach, while the new involutive negation operator is proposed herein. Further, we have studied the validity of Boolean laws in the proposed approach, with the special attention to laws of excluded middle and contradiction. It is proven that these laws are valid in LBIFS-IBA approach.

The aim of future work is to evaluate the applicative value of LBIFS with IBA. One of the directions is to extend IBA logical aggregation operator to deal with LBIFS. Furthermore, we aim to define similarity measure for LBIFS and compare it with classical IFS similarities on various clustering and pattern recognition problem.

\section{References}

[1] K. Atanassov, Intuitionistic fuzzy sets, Fuzzy Sets and Systems 20 (1986) 87-96.

[2] K. Atanassov, Intuitionistic fuzzy sets: past, present and future, in: M. Wagenknecht, R. Hampel (Eds.), Proc. 3rd EUSFLAT Conference, Zittau, 2003, pp. 12-19.

[3] K. Atanassov, On Intuitionistic Fuzzy Sets Theory, Studies in Fuzziness and Soft Computing 283, Springer, Berlin, 2012.

[4] K. Atanassov, G. Gargov, Interval valued intuitionistic fuzzy sets, Fuzzy Sets and Systems, 31(3) (1989) 343-349.

[5] H. Bustince, P. Burillo, Vague sets are intuitionistic fuzzy sets, Fuzzy Sets and Systems 79(3) (1996) 403-405.

[6] B. C. Cuong, P. L. Lanzi, N. T. Thong, A novel intuitionistic fuzzy clustering method for geodemographic analysis, Expert Systems with Applications 39(10) (2012) 9848-9859.

[7] I. Despi, D. Opris, E. Yalcin, Generalised Atanassov Intuitionistic Fuzzy Sets, in: D. Malzahn (Ed.), eKNOW 2013: The Fifth International Conference on Information, 
Process, and Knowledge Management, Nice, 2013, pp. 51-56.

[8] I. Dragović, N. Turajlić, D. Radojević, B. Petrović, Combining Boolean consistent fuzzy logic and AHP illustrated on the web service selection problem, International Journal of Computational Intelligence Systems 7(supp. 1) (2014) 84-93.

[9] D. Dubois, S. Gottwald, P. Hajek, J. Kacprzyk, H. Prade, Terminological difficulties in fuzzy set theory - The case of "Intuitionistic Fuzzy Sets", Fuzzy Sets and Systems 156(3) (2005) 485-491.

[10] E. B. Jamkhaneh, S. Nadarajah, A New generalized intuitionistic fuzzy sets, Hacettepe Journal of Mathematics and Statistics 10 (2014). doi: 10.15672/HJMS. 2014367557

[11] F. Jin, L. Pei, H. Chen, L. Zhou, Interval-valued intuitionistic fuzzy continuous weighted entropy and its application to multi-criteria fuzzy group decision making, Knowledge-Based Systems 59 (2014) 132-141.

[12] K. P. Lin, A novel evolutionary kernel intuitionistic fuzzy C-means clustering algorithm, IEEE Transactions on Fuzzy Systems, 22(5) (2014) 1074-1087.

[13] H. C. Liu, Liu's generalized intuitionistic fuzzy sets, Journal of Educational Measurement and Statistics 18(1) (2010) 1-14.

[14] P. Milošević, B. Petrović, V. Jeremić, IFS-IBA similarity measure in machine learning algorithms, Expert Systems with Application 89 (2017) 296-305.

[15] P. Milošević, A. Poledica, A. Rakićević, V. Dobrić, B. Petrović, D. Radojević, IBA-based framework for modeling similarity, International Journal of Computational Intelligence Systems 11(1) (2018) 206-218.

[16] P. Milošević, A. Poledica, A. Rakićević, B. Petrović, D. Radojević, Introducing interpolative Boolean algebra into intuitionistic fuzzy sets, in: J. M. Alonso, H. Bustince, M. Reformat (Eds.), Proc. 9th EUSFLAT Conference, Gijon, 2015, pp. 1389-1394.

[17] T. K. Mondal, S. Samanta, Generalized intuitionistic fuzzy sets, Journal of Fuzzy Mathematics 10(4) (2002) 839-862.

[18] J. H. Park, J. Hwang, J. Kim, B. Park, J. Park, J. Son, S. Lee, Similarity measure between generalized intuitionistic fuzzy sets and its application to pattern recognition, Journal of Computational Analysis \& Applications 20(1) (2016) 984-994.

[19] D. Radojević, [0, 1]-valued logic: A natural generalization of Boolean logic, Yugoslav Journal of Operations Research 10(2) (2000) 185-216.

[20] D. Radojević, Fuzzy Set Theory in Boolean Frame, International Journal of Computers, Communications \& Control 3(3) (2008) 121131.

[21] D. Radojević, Logical aggregation based on interpolative, Mathware \& Soft Computing, 15(1) (2008) 125-141.

[22] R. Srinivasan, N. Palaniappan, Some operators on intuitionistic fuzzy sets of root type, Annals of Fuzzy Mathematics and Informatics 4(2) (2012) 377-383.

[23] I. K. Vlachos, G. D. Sergiadis, Intuitionistic fuzzy information-applications to pattern recognition, Pattern Recognition Letters 28(2) (2007) 197-206.

[24] Xu, Z., \& Zhao, N. Information fusion for intuitionistic fuzzy decision making: an overview, Information Fusion 28 (2016) 10-23. 